\title{
Fabrication of Soft-Glass-Based Wire Array Metamaterial Fibers for Applications at Infrared Frequencies
}

Hayash, Juliano G.; Lwin, Richard; Stefani, Alessio ; Fleming, Simon; Kuhlmey, Boris T.; Argyros, Alexander

Published in:

Journal of Lightwave Technology

Link to article, DOI:

10.1109/jt.2019.2926808

Publication date:

2019

Document Version

Peer reviewed version

Link back to DTU Orbit

Citation (APA):

Hayash, J. G., Lwin, R., Stefani, A., Fleming, S., Kuhlmey, B. T., \& Argyros, A. (2019). Fabrication of Soft-GlassBased Wire Array Metamaterial Fibers for Applications at Infrared Frequencies. Journal of Lightwave Technology, 37(19), 5001-5009. https://doi.org/10.1109/jt.2019.2926808

\section{General rights}

Copyright and moral rights for the publications made accessible in the public portal are retained by the authors and/or other copyright owners and it is a condition of accessing publications that users recognise and abide by the legal requirements associated with these rights.

- Users may download and print one copy of any publication from the public portal for the purpose of private study or research.

- You may not further distribute the material or use it for any profit-making activity or commercial gain

- You may freely distribute the URL identifying the publication in the public portal 


\title{
Fabrication of soft-glass based wire array metamaterial fibers for applications at infrared frequencies
}

\author{
Juliano G. Hayashi, Richard Lwin, Alessio Stefani, Simon Fleming, Boris T. Kuhlmey, and Alexander \\ Argyros
}

\begin{abstract}
Metamaterials for the mid-infrared spectrum require subwavelength meta-structures with dimensions of a few hundreds of nanometers. Fabrication via fiber drawing is challenging as the Plateau-Rayleigh instability caused by interfacial surface tension between the liquid metal and the dielectric during the drawing leads to fluctuation of the structure, preventing drawing of uniform wire array structures with such dimensions. Here, conventional fiber drawing technique is employed in the fabrication of wire array metamaterial fibers containing tin wires embedded in soda-lime glass. PlateauRayleigh instabilities ensuing detrimental deformations on submicron metallic structures are minimized through the selection of materials with favorable rheological properties and the optimization of the drawing parameters. Uniform wire array structures with wire diameter and spacing as small as $143 \mathbf{~ n m}$ and $286 \mathrm{~nm}$, respectively are demonstrated. The application of this established fabrication process represents a large-volume and low-cost alternative for the production of hyperbolic metamaterials. The new metamaterial fibers achieved open up a range of exciting applications at mid-infrared frequencies, such as lifetime engineering and super-resolution imaging.
\end{abstract}

Index Terms - Fiber fabrication, metamaterial fiber, PlateauRayleigh instability, wire array medium.

\section{INTRODUCTION}

$\mathrm{M}$ ETAMATERIAls are artificial composite materials that exhibit exotic properties due to their components and subwavelength structure [1]. During the past decades, several new materials have emerged from this research field, resulting in the development of extraordinary new metadevices such as metalenses [2], metasurfaces [3], hyperlenses [4], [5], and invisibility cloaks [6]. Despite the improvement of the fabrication methods over the years, the production of certain

This paragraph of the first footnote will contain the date on which you submitted your paper for review.

The authors are with the Institute of Photonics and Optical Science (IPOS), School of Physics, University of Sydney, Sydney, NSW, 2006, Australia (email: jgri9078@uni.sydney.edu.au; richard.lwin@sydney.edu.au; alessio.stefani@sydney.edu.au;

boris.kuhlmey@sydney.edu.au and alexander.argyros@sydney.edu.au).

Alessio Stefani is also with DTU Fotonik, Department of Photonics Engineering, Technical University of Denmark, DK-2800 Kgs. Lyngby, Denmark. types of meta-structures, such as tapered wire arrays (hyperlenses) remains a challenge. Fiber drawing is an attractive alternative to produce such tapered wire structures, and also represents a low-cost and high volume method in comparison with conventional fabrication techniques such as lithography: The drawing process can be used to scale down large preforms to the micro- and nano-scale, allowing large volume production, which reduces the cost. However, the presence of metal in the fiber's structure, required to achieve the most common metamaterials, introduces additional constraints and challenges in the co-drawing process [7], [8]. From the fabrication perspective, the materials selected for this type of multimaterial fibers must be chemically compatible (so no chemical reactions such as oxidation occur) and have a favorable combination of rheological properties. Even then, the drawing parameters must be optimized to keep the metallic structure relatively uniform in the resultant fiber.

Recently, hyperbolic metamaterial fibers based on polymer and indium have been demonstrated with split-ring [9], [10], and wire array meta-structures [11], [12], [13], with applications including sub-diffraction imaging [13]. However, the Plateau-Rayleigh instability [14], [15], [16], which is the tendency of a liquid column to break into droplets, limits the drawn metal/dielectric meta-structure to a minimum stable size which depends on the rheological properties of the components and the drawing parameters [12], [17]. Even with optimized drawing parameters, the Plateau-Rayleigh instability limits the wire diameter and spacing to $\simeq 2 \mu \mathrm{m}$ in the polymer/indium system [12], [17]. Considering that metamaterials must have an elementary meta-structure smaller than 1/10th of the desired operational wavelength, the operational range of the polymer-based metamaterial fibers is limited to frequencies in the microwave/ $\mathrm{THz}$ spectrum [13] and the far-infrared [12].

Wire array metamaterials and hyperlenses (tapered wire arrays) for operation at mid-infrared frequencies (MIR) require meta-structures at a scale of a few hundreds of nanometers. Thus, the fabrication of such metamaterial fibers is only feasible using alternative combination of materials with a Plateau-Rayleigh instability imposed limit below these dimensions. Example of drawing of submicron metal wires in different contexts can be found in the literature using glass as the dielectric: Taylor has demonstrated the drawing of single- 
submicron wires of different metals embedded in a variety of glasses, an approach called the Taylor wire process, achieving continuous antimony wires with a diameter as small as $200 \mathrm{~nm}$ [18]. Zhang reported the drawing of copper/phosphorus alloy wires embedded in borosilicate glass by several consecutive draw-cut-stack-draw processes [19]. Similarly, Badinter has fabricated a densely-packed bundle of bismuth and lead/tin alloy nanowires embedded in borosilicate glass [20], producing wire dimeters as small as $50 \mathrm{~nm}$ over a $1 \mathrm{~m}$ length. For wire array metamaterial fibers, a consistent quality of the drawn subwavelength meta-structure is necessary but not sufficient: The materials must also be selected for the optical performance of the resultant metamaterial. Recently, a systematic study of simulated optical losses in wire arrays made of material pairs that should lead to smaller instabilityinduced size limits suggested that tin wires in soda-lime glass could be suitable for imaging applications at mid-infrared frequencies [21]. In this scenario, the optical loss of the metamaterial is dominated by the high absorption of the submicron metallic wires [21], which can be several orders of magnitude higher than the absorption of the dielectric. Thus, the MIR metadevices must be limited to a few hundred microns in length to restrict their losses to feasible levels.

In this work, we demonstrate the fabrication of soft-glass based wire array metamaterial fibers, containing approximately five hundred tin wires with diameters of a few hundred nanometers hexagonally arranged inside a soda-lime fiber. The Plateau-Rayleigh instability is characterized in such a system for fiber drawing with optimized parameters. The fiber tension during the drawing process is optimized to maximize the suppression of the Plateau-Rayleigh instability, resulting in the fabrication of highly uniform wire array structures even for averaged wire diameter as small as $143 \mathrm{~nm}$. The established fabrication process represents a low-cost and large volume production alternative to the usual lithographybased planar techniques for hyperbolic metamaterials with operation wavelength as small as the mid-infrared. Furthermore, the new drawn wire array metamaterial fibers and preforms offer the possibility to fabricate tapered structures, realizing wire array magnifying hyperlenses for subdiffraction imaging with large magnification [5], [13], in the MIR, which are impractical to fabricate with conventional planar techniques.

\section{PlateAU-RAYLEIGH INSTABILITY IN MULTIMATERIAL}

\section{DRAWING}

Ideally, during the drawing of a preform formed by a metallic structure embedded in a dielectric matrix, the metal is liquid, and the dielectric has a dynamic viscosity within the drawable range [7], [8]. In this scenario, the multimaterial preform is a complex fluid dynamic system under competing forces [22]. The surface tension, which tends to minimize the surface area of the inner material, competes with inertial and viscous forces, which tend to maintain the cylindrical shape of the system. If this interfacial surface tension between the materials dominates, it causes any small fluctuations in the liquid metal column to grow over time, a phenomenon known as the Plateau-Rayleigh instability [14], [15], [17]. Such perturbations growing exponentially with time can significantly deform the metal/dielectric structure and eventually break the inner liquid metal into separate droplets.

Following Tomotika's model for the growth of the instability [14], [15], the periodic perturbation in radius for an incompressible fluid cylinder surrounded by an unbounded viscous fluid will grow over time $(t)$ and along the axial length $(z)$ as,

$$
R(z, t)=R_{0}+A_{0} e^{g \cdot t} \cos (k z)
$$

where $R_{0}$ is the initial cylinder's radius, $A_{0}$ is the initial amplitude, and $k$ is the wavenumber of the perturbation. The growth of the perturbation is dictated by the gain term, $g$, defined by [14], [15], [17],

$$
g=\frac{\sigma}{2 R \mu}\left(1-x^{2}\right) \cdot \phi\left(x, \mu^{\prime} / \mu\right)
$$

where $\sigma$ is the interfacial surface tension between the inner fluid cylinder and the unbound surrounding viscous fluid, $R$ is the radius of the perturbed inner cylinder, and $x=k R$. The term $\mu$ is the viscosity of the unbounded surrounding viscous fluid, $\mu^{\prime}$ is the viscosity of the inner fluid cylinder, and $\phi$ is a function composed of Bessel functions that can be found in reference [14]. For liquid indium wires embedded in PMMA fibers at the typical drawing temperature $\left(\simeq 200^{\circ} \mathrm{C}\right.$ ), the viscosities are $\mu^{\prime} \sim 10^{-3}$ Pa.s [23] and $\mu \sim 10^{5}$ Pa.s [24], respectively, which results in $\mu^{\prime} / \mu \sim 10^{-8}$. In such regime and considering only small perturbations in amplitude relative to the radius of the liquid metal wire, no particular spatial frequency dominates and the perturbations evolve independently [12], [17]. For small values of $x$, the function $\left(1-x^{2}\right) \cdot \phi\left(x, \mu^{\prime} / \mu\right)$ can be approximated to 1 , which means that all perturbations with long wavelength possess similar gain. Equation (2) can then be simplified to [12], [17],

$$
g \approx \frac{\sigma}{2 R \mu}
$$

For liquid tin wires embedded in soda-lime glass fiber at their typical drawing temperature $\left(\simeq 700^{\circ} \mathrm{C}\right)$, the same behavior and approximation hold as $\mu^{\prime} / \mu \sim 10^{-10}$ ( $\mu^{\prime} \sim 10^{-3}$ Pa.s for tin [25] and $\mu \sim 10^{7}$ Pa.s for soda-lime [26]). In the multimaterial drawing context, (3) means that the instabilities grow more rapidly for smaller wires, for a smaller viscosity of the external dielectric, and for larger interfacial surface tension between the materials.

Assuming that the interfacial surface tension between the liquid metal and the dielectric during the drawing is dominated by the surface tension of the metal, the replacement of the 
selected system from indium/PMMA to tin/soda-lime does not change considerably the gain term $g$. Even considering their different drawing temperatures, $\simeq 200^{\circ} \mathrm{C}$ for the indium/polymer system and $\simeq 700^{\circ} \mathrm{C}$ for tin/soda-lime system, both metals exhibit a surface tension in the order of $0.5 \mathrm{~N} / \mathrm{m}$ [27], [28], [29]. Further characterization of the interfacial surface tension between the materials in their respective drawing temperatures is required for a more accurate analysis.

On the other hand, the replacement of the PMMA with soda-lime in wire array preforms can increase the highest drawable viscosity of the dielectric by one order of magnitude, from $\mu \sim 10^{6}$ Pa.s for PMMA [24] to $\mu \sim 10^{7}$ Pa.s for sodalime [26]. These limits depend on the tensile strength of the wire array preforms and were established by several test drawings with both material combinations minimizing the drawing temperature $\left(\simeq 170^{\circ} \mathrm{C}\right.$ for $\mathrm{PMMA} /$ indium and $\simeq 680^{\circ} \mathrm{C}$ for the tin/soda-lime preforms). Based on (3), this could lead to a reduction of the gain term $g$ by one order of magnitude. However, recent work has demonstrated that the instabilities in the drawing of wire array fibers with PMMA and indium are affected by other fabrication parameters besides the drawing temperature/viscosity, such as feed/drawing velocities, and neck-down profile of the preform [17]. A similar behavior is expected in the tin/soda-lime system, thus a comparison between viscosities alone is unlikely to predict accurately the impact of the change of materials in the instabilities.

According to (1), besides the optimization of the gain term $g$, another alternative to suppress the growth of the PlateauRayleigh instability is to minimize the time the fiber spends in a viscous state during the drawing (dwell time). This is possible by performing faster drawings and quenching the fiber as close as possible to the end of the neck-down region [20], [30], [31]. However, this is not trivial due to the typical timescale over which instabilities grow, which requires a pulling rate at the $\mathrm{m} / \mathrm{s}$ scale [19], [31], and a quenching with water a few millimeters below the neck-down region [20], [30], [31], which is not feasible in some furnaces. This method is also limited because a substantial growth in the instabilities can occur before the end of the draw (neck-down region) [17].

\section{METAMATERIAL FIBER FABRICATION}

Fabrication is similar to that used for photonic crystal fibers [32], [33]. When glasses are selected as the dielectric material for the fibers and commercial tubes are available in a range of dimensions at reasonable prices (which is the case for sodalime glasses), the stack-and-draw technique is preferred due to its high flexibility [32], [33]. For the fabrication of hyperbolic metal/dielectric metamaterial fibers, this technique must be adapted to include the metallic structure and avoid its deformation/fluctuations due to Plateau-Rayleigh instabilities.

The fabrication process established here is divided into the following two steps: the fabrication of the metamaterial preform, which is a macroscopic version of the final metamaterial fiber, and the drawing of the preform into a fiber. Below, the fabrication of a tin/soda-lime wire array metamaterial fiber composed of approximately five hundred tin wires with $d / \Lambda=0.5$ and $d$ around a few hundred nanometers is fully described. Such a structure is intended for imaging applications and lifetime engineering in the midinfrared [21], [34].

\section{A. Metamaterial preform fabrication}

Soda-lime capillaries are drawn in a fiber tower from a large soda-lime tube, as illustrated in Fig. 1(a). The original tube is heated around its softening point $\left(\mathrm{T}=720^{\circ} \mathrm{C}\right.$, corresponding to a viscosity of $10^{6.6} \mathrm{~Pa} . \mathrm{s}$ [35]), allowed to drop under gravity, and attached to the pulling system of the tower. The desired diameter reduction is achieved by adjusting the feed $\left(\mathrm{v}_{\text {feed }}\right)$ and drawing velocities $\left(\mathrm{v}_{\text {drawing }}\right)$. The resultant capillaries are stacked in a hexagonal pack with an adjustable 3D printed hexagonal stacker (Fig. 1(b)) and inserted inside a large soda-lime tube, called a jacket. The selected jacket must have an inner diameter matching the size of the hexagonal pack. The remaining air gaps are filled with solid soda-lime rod supports to avoid structural deformations, forming the stacked preform (Fig. 1(c)). If a circular rather than hexagonal region of holes is desired, the solid rod supports on the outer boundary can be replaced by corresponding hollow capillaries.

The hole array cane is fabricated in the fiber tower by stretching the stacked preform with vacuum applied between the capillaries to collapse the interstitial air gaps. Figure 1(d)

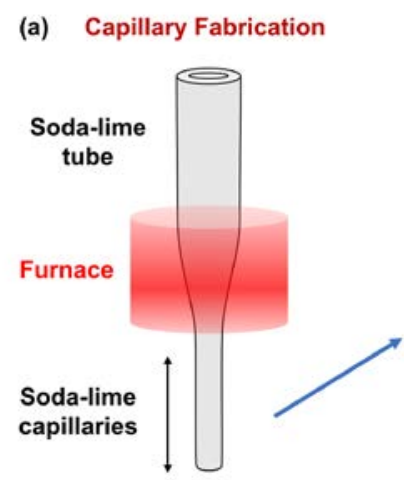

(d)

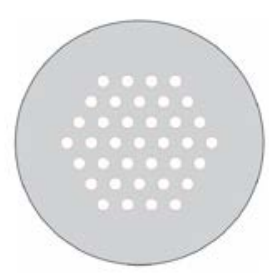

Hole array cane (b) Hexagonal Stacking

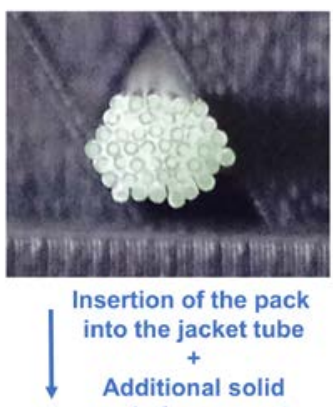

(c)

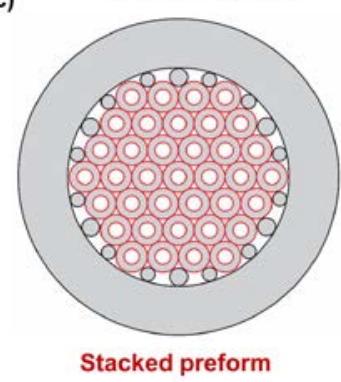

Fig 1. Schematic of the hole array cane fabrication. (a) Drawing of soda-lime tubes into smaller capillaries in the fiber tower. (b) Stacking of the capillaries in a hexagonal pack. (c) Insertion of the hexagonal pack into another sodalime tube (jacket). Additional solid rod supports are employed to fill the outer air gaps, forming the stacked preform. (d) The stacked preform is stretched in the fiber tower with vacuum applied between the capillaries to collapse the interstitial air gaps, generating the hole array cane. 

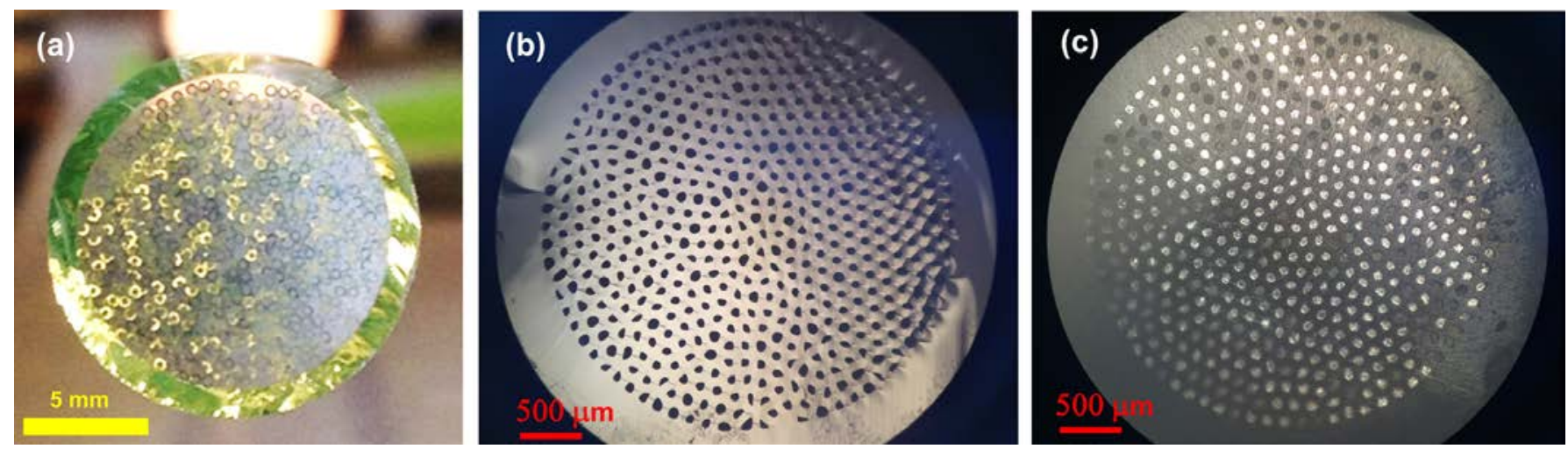

Fig. 2. (a) Stacked preform after the insertion of the stacked pack into the jacket tube. (b) Resultant hole array cane after drawing. (c) Wire array cane obtained by filling the hole array cane with a pressure-assisted filling technique. Tin wires with $d_{\text {avg }} \simeq 75 \mu \mathrm{m}$ and $\Lambda \simeq 150 \mu \mathrm{m}$.

shows a schematic of a resultant hole array cane, showing no air gaps and a slight reduction in the outside diameter of the overall structure.

Figure 2(a) shows an image of the stacked preform fabricated for our metamaterial fiber, containing around five hundred soda-lime capillaries with outside diameter (OD) of $500 \mu \mathrm{m}$ and inner diameter (ID) of $250 \mu \mathrm{m}$, introduced inside a soda-lime jacket tube with $\mathrm{OD}=12 \mathrm{~mm}$ and $\mathrm{ID}=10 \mathrm{~mm}$. Figure 2(b) shows the resulting hole array cane after the stretching of the stacked preform, containing holes with average diameter $\left(d_{\text {avg }}\right)$ around $75 \mu \mathrm{m}$, hole spacing $(\Lambda)$ around $150 \mu \mathrm{m}$, and overall OD around $4 \mathrm{~mm}$. The deformations in the structure observed in Fig. 2(b) are caused by imperfections in the stacking pack, which arise from the high flexibility and small size of the employed capillaries $(\mathrm{OD}=500 \mu \mathrm{m})$. Static forces make a perfect stacking impractical, and there is a small reorganization of the capillaries when the whole hexagonal pack is inserted in the jacket tube due to its high flexibility.

After the fabrication of the hole array cane, its air holes are filled with tin using a pressure-assisted filling technique, similarly as employed in [36], [37], resulting in a wire array cane. In this filling technique, one side of the cane is immersed into a pool of liquid metal contained inside a resistance oven at a temperature of $280^{\circ} \mathrm{C}$. The other side of the cane, kept about two centimeters outside the oven and at room temperature, is attached to a vacuum system formed by a vacuum pump, a leaking valve to control the pressure, and a connecting hose. The partial vacuum slowly generated inside the holes of the cane induces a negative pressure in comparison to the surface of the liquid metal pool. The pressure difference, combined with capillary forces, draws up the liquid tin through the cane until the top of the liquid columns reaches the outside of the oven and solidifies. For a cane with a length of $50 \mathrm{~cm}$, based on surface tension the filling time can be estimated for tin into holes with $d_{\text {avg }} \simeq 100 \mu \mathrm{m}$ to be around $10 \mathrm{~s}$ [34]. After this period, the oven's temperature is slowly decreased to room temperature, the vacuum pump is turned off, and the filled hole array is removed from the oven.

Figure 2(c) shows the wire array cane achieved after the filling of the hole array cane seen in Fig. 2(b). Note that the bright regions are the tin wires, while the dark regions indicate that the filling of some of the holes was not completely successful (the molten metal column has not reached the top of the hole array cane). This problem likely arises from the blocking of these holes by oxidation residue or other dust fragments. However, such samples can still be used if a substantial length of the hole array cane is completely filled with metal and the defective region is discarded. The typical filling length achieved in our system was $\simeq 50 \mathrm{~cm}$, limited by the vertical size of our oven.

After its filling, the wire array cane is introduced inside a soda-lime jacket tube and stretched to smaller dimensions in a fiber tower, a process referred to here as sleeving and stretching. Vacuum is applied in the small gap between the cane and the jacket tube during the drawing, resulting in a scaled-down wire array structure with an extra layer of glass added to its outside. The typical draw ratio employed in this process varies between 5 and 10x, depending on the size of the jacket tube and the intended outside diameter for the stretched sample. Since this stretching process involves the co-drawing of metal and glass, care must be taken with the selected drawing temperature and the final diameter of the wires. Previous work reports the metallic structure starts to fluctuate significantly for wire diameters around $5 \mu \mathrm{m}$ for the indium/PMMA system [12], [17], and below $1 \mu \mathrm{m}$ for metallic wires embedded in glass [31]. Therefore, to avoid instabilities in this first co-drawing, the final size of the wires in this stage should not be smaller than $5 \mu \mathrm{m}$, and high drawing temperatures must be avoided. This scaled-down version of the wire array cane with wires larger than $5 \mu \mathrm{m}$ is called here the metamaterial preform.

Figure 3(a) shows a micrograph of a metamaterial preform containing a few hundred wires, obtained with the sleeving and stretching process of the filled wire array cane seen in Fig. 2(c), performed under favorable conditions to avoid PlateauRayleigh instabilities: low drawing temperature $\left(\mathrm{T} \simeq 680^{\circ} \mathrm{C}\right.$ ) 

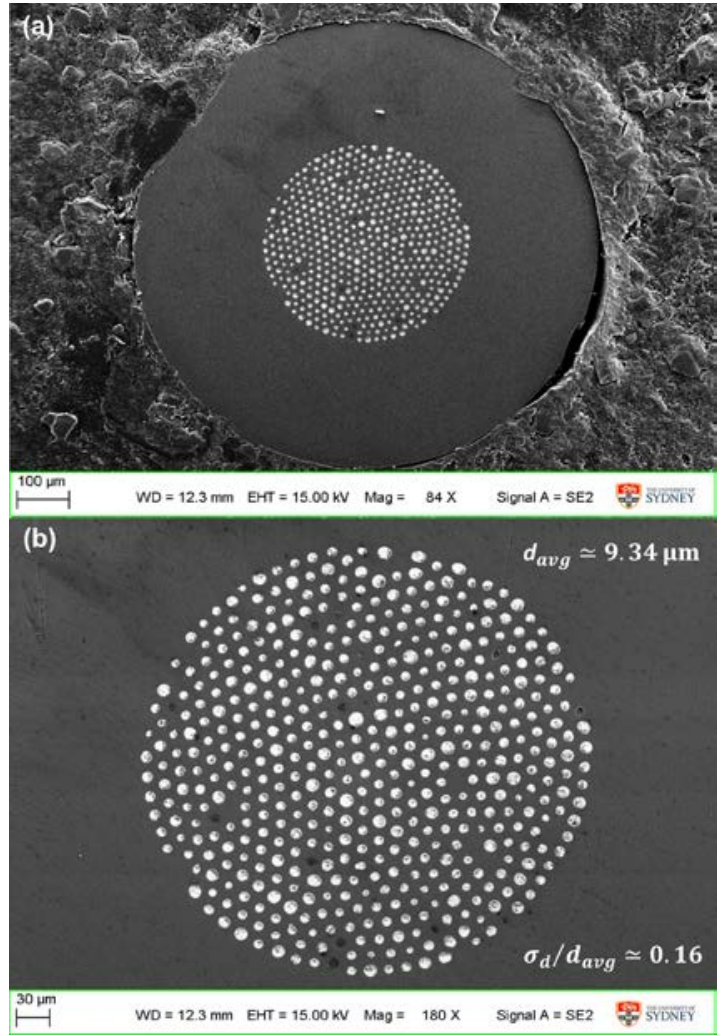

Fig. 3. (a) Metamaterial preform fabricated by the sleeving and stretching of the wire array cane (Fig. 2(c)) with $\mathrm{OD} \simeq 900 \mu \mathrm{m}$. (b) close-up of the wire array region, showing $d_{\text {avg }} \simeq 9.34 \mu \mathrm{m}, \Lambda \simeq 18 \mu \mathrm{m}$ and $\sigma_{d} / d_{\text {avg }} \simeq 0.16$.

and high pulling tension. Figure 3(b) shows a zoomed pictured of the wire array region, with $d_{\text {avg }} \simeq 9.34 \mu \mathrm{m}$ and $\Lambda \simeq 18 \mu \mathrm{m}$.

With a high-resolution cross-section micrograph, like the one presented in Fig. 3, the fluctuation of the wire diameter in the structure can be quantified by calculating the term $\sigma_{d} / d_{\text {avg }}$, where $\sigma_{d}$ is the standard deviation of the wire diameter distribution and $d_{\text {avg }}$ is the average wire diameter. The metamaterial preform shown in Fig. 3 has a $\sigma_{d} / d_{\text {avg }} \simeq 0.16$, which represents a small fluctuation in the metallic structure. Note that the hole array cane after the filling, shown in Fig. 2(c) , already presents a small degree of fluctuation in its holes due to imperfections in the stacking ( $\sigma_{d} / d_{\text {avg }} \simeq 0.13$ ), indicating that the first co-drawing of metal and glass (sleeving and stretching) did not create a significantly increase the degree of diameter fluctuations. Such a condition is essential to achieve a uniform structure in the second and final stretching process.

Note that, because some holes/wires of the characterized samples are not perfectly circular and they were manually measured, the terms $d_{\text {avg }}$ and $\sigma_{d}$ calculated throughout this paper should be treated as an approximation.

\section{B. Drawing of the preform to fiber}

The final metamaterial fiber is fabricated by another sleeving and stretching of the metamaterial preform, where the wires are scaled down from $d_{\text {avg }} \simeq 10 \mu \mathrm{m}$ to a few hundred nanometers. Due to the exponential growth of the PlateauRayleigh instability, the quality of the drawn metallic structure is very sensitive to the drawing parameters such as temperature, pulling tension, feeding and drawing velocities.

According to (3), the instabilities in the metallic wires grow more rapidly for a lower viscosity of the dielectric matrix. Consequently, these fluctuations can be minimized if the drawing process is performed with the highest possible viscosity of the dielectric matrix, i.e., at the minimum drawing temperature corresponding to highest pulling tension before the preform or fiber breaks. For a specific size of preform and draw ratio, such maximum drawing tension is determined by the tensile strength of the preform, which is diminished by surface imperfection/defects on the glass.

Figure 4(a) and Fig. 4(b) show examples of micrographs of two different wire array metamaterial fibers drawn on the limit of the pulling tension, fabricated by sleeving and stretching the metamaterial preform seen in Fig. 3 with a jacket tube with $\mathrm{OD}=10 \mathrm{~mm}, \mathrm{ID}=1.2 \mathrm{~mm}$ and $\mathrm{v}_{\text {feed }}=5 \mathrm{~mm} / \mathrm{min}$. These fibers contain $d_{\text {avg }}=418 \mathrm{~nm} \quad\left(\sigma_{\mathrm{d}} / \mathrm{d}_{\text {avg }} \simeq 0.57\right) \quad$ and $d_{\text {avg }}=774 \mathrm{~nm} \quad\left(\sigma_{\mathrm{d}} / \mathrm{d}_{\text {avg }} \simeq 0.22\right), \quad$ OD $\quad$ of $\simeq 400 \mu \mathrm{m}$ $\left(\mathrm{v}_{\text {drawing }} \simeq 6 \mathrm{~m} / \mathrm{min}\right)$ and $\simeq 800 \mu \mathrm{m}\left(\mathrm{v}_{\text {drawing }} \simeq 3 \mathrm{~m} / \mathrm{min}\right)$, and drawing ratio of $\simeq 25 \mathrm{x}$ and $\simeq 13 \mathrm{x}$, respectively. Many different experiments were carried out with several preforms and different drawing ratios to study the instability. For every fiber with different draw ratio, the drawing parameters were adjusted to achieve values close to the experimental maximum drawing tension (determined by trial and error based on breakage) and, consequently, close to the minimum drawing temperature. Since the fiber tower does not have a system to measure the pulling tension, the values employed when drawing must be considered experimental approximations to the maximum feasible drawing tension. Note that the black regions consist of empty holes or wires that had their top extremities removed in the cleaving process.

Figure 4(c) shows a plot of the instability term $\sigma_{d} / d_{a v g}$ as a function of $d_{\text {avg }}$ for wire array metamaterials fibers fabricated at the limit of the pulling tension. The red dots correspond to the different tin/soda-lime wire array fibers with a few hundred wires (Fig. 4(a) and Fig. 4(b)), while the black dots correspond to a different set of fibers with an equivalent tin/soda-lime structure with a few tens of wires $(\simeq 50)$. The points with $d_{\text {avg }} \simeq 10 \mu \mathrm{m}$ are the respective preforms, and the curves correspond to nonlinear fittings of the function $\alpha e^{\beta / d}$ (free parameters $\alpha$ and $\beta$ ), which is the expected form according to the standard deviation of (1) [12]. For comparison, the plot also contains the instability term of wire array metamaterial fibers fabricated with the indium/PMMA system (blue dots), reported in reference [12]. As expected, the 

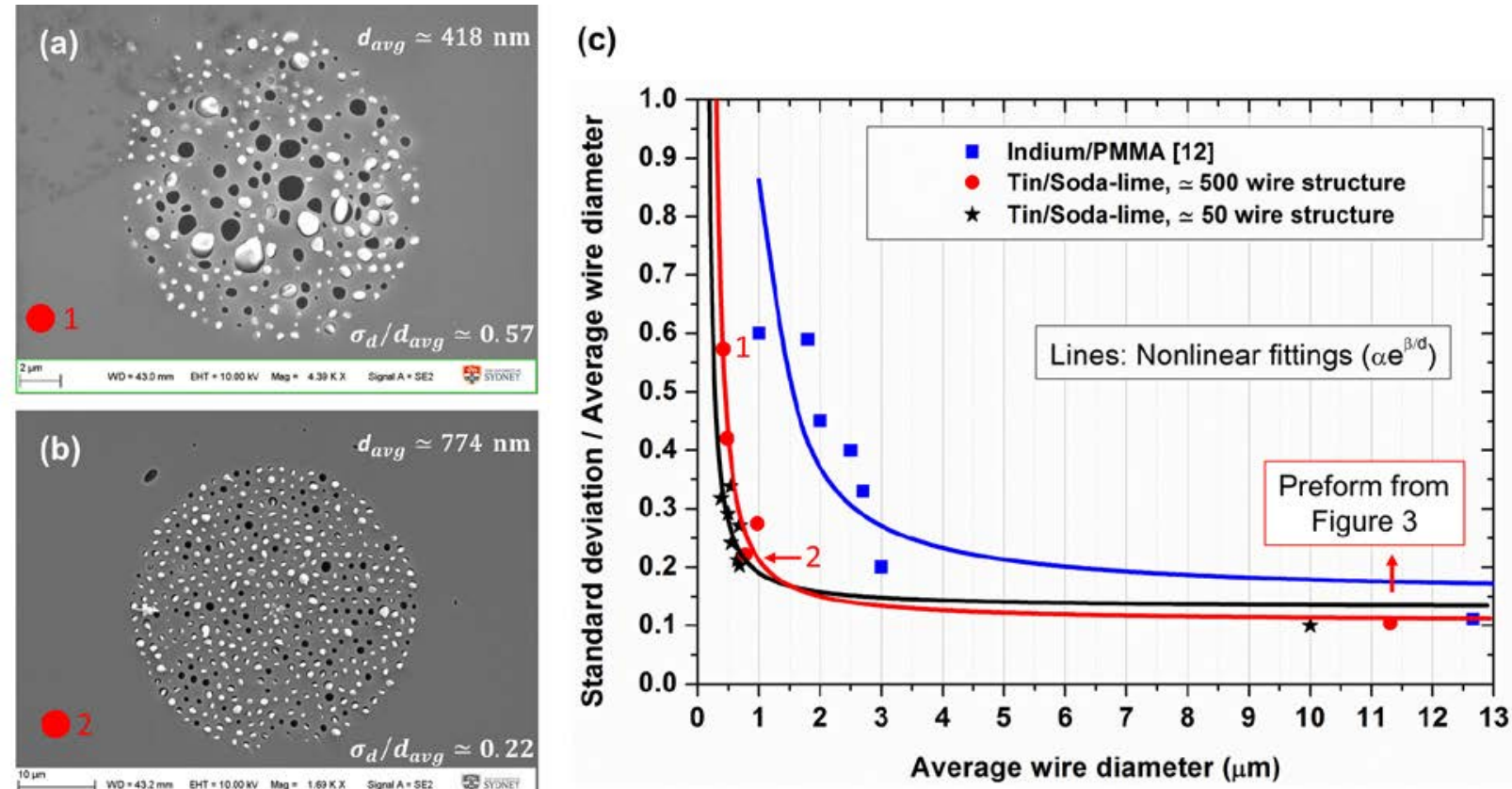

Fig. 4. Examples of tin/soda-lime wire array metamaterial fibers drawn under the limit of tension from the metamaterial preform from Fig. 3: (a) $d_{\text {avg }} \simeq 418$ $\mathrm{nm}, \quad \sigma_{d} / d_{\text {avg }} \simeq 0.57 ; \quad$ (b) $d_{\text {avg }} \simeq 774 \mathrm{~nm}, \sigma_{d} / d_{\text {avg }} \simeq 0.22$. (c) Comparison of the instability term $\sigma_{d} / d_{\text {avg }}$ as a function of the $d_{\text {avg }}$ between the indium/PMMA system (blue dots - extracted from reference [12]) and the tin/soda-lime system for $\simeq 500$ wires (red dots) and $\simeq 50$ wires (black dots) structures. The curves correspond to nonlinear fittings of the function $\alpha e^{\beta / d}$, which is the expected form according to the standard deviation of (1) [12].

fluctuation in the wire array structures increases for decreasing $d_{\text {avg }}$ in all cases.

Considering the Plateau-Instability limit as the wire diameter corresponding to $\sigma_{d} / d_{\text {avg }}=1 / e \simeq 0.37$, Fig. 4(c) shows that this standard for drawn structures shifts from a wire diameter around $2 \mu \mathrm{m}$ for the indium/PMMA system, to a wire diameter around $\simeq 550 \mathrm{~nm}(\simeq 3.6 \mathrm{x}$ smaller $)$ and $\simeq 360 \mathrm{~nm}(\simeq 5.5 \mathrm{x}$ smaller $)$ for the tin/soda-lime fibers with $\simeq 500$ (red line) and $\simeq 50$ (black line) wires, respectively. As discussed in the previous section, the replacement of the polymer by glass increases the maximum drawable viscosity of the dielectric of wire array preforms by one order of magnitude, which could lead to an equivalent reduction in the Plateau-Instability limit. The smaller improvement seen in the structures of Fig. 4 indicates that other parameters that affects the instabilities are limiting its suppression (feed and drawing velocities, neck-down shape, and dwell time of the fiber in the hot zone region). In Section C, a new fabrication process is proposed and employed to further improve the maximization of the dielectric viscosity and also address the influence of those other parameters in the Plateau-Rayleigh instability.

Note that small fluctuations in the wire diameters are already seen in the metamaterial preforms before the last drawing (Fig. 3), which are due to inconsistent capillary sizes employed in the hole array stacking or appearing during the hole array cane drawing due to asymmetries and vacuum (they are not from Plateau-Rayleigh instability). This type of structural fluctuation varies between the different preforms used to fabricate the different fibers. Therefore, the PlateauRayleigh instability limit shown in Fig. 4(c) should be treated as an approximation.

\section{Stretching under extreme conditions}

In theory, the allowed pulling tension in a drawing/stretching process depends on the tensile strength of the fiber, which is strongly related to the quality of the glass surface. For soda-lime glass, such tensile strength can vary from 20 to $200 \mathrm{MPa}$ ( $\simeq$ from 2 to $20 \mathrm{~kg} / \mathrm{mm}^{2}$ [26]). In practice, when a continuous drawing is employed, maintaining a high pulling tension close to the maximum is unfeasible because some defect on the surface of the glass eventually breaks the fibers.

Here, we propose the replacement of the continuous drawing in the last stretching of the metamaterial preform by a high-tension localized stretching process. In this scenario, because only a small portion of the preform is heated and stretched, a pulling tension per area close to the maximum ( $\simeq 20 \mathrm{~kg} / \mathrm{mm}^{2}$ for soda-lime) is feasible if the selected heated region possesses a good surface quality. Consequently, the higher pulling tension leads to a stretching of the preform with a higher viscosity of the dielectric, which results in further suppression of the Plateau-Rayleigh instability. This new approach reduces the time that the fiber spends in a viscous state during stretching, which can lead to a further reduction of the instabilities depending on the time scale of the quenching. The smaller hot zone also affects the profile of the neck-down 


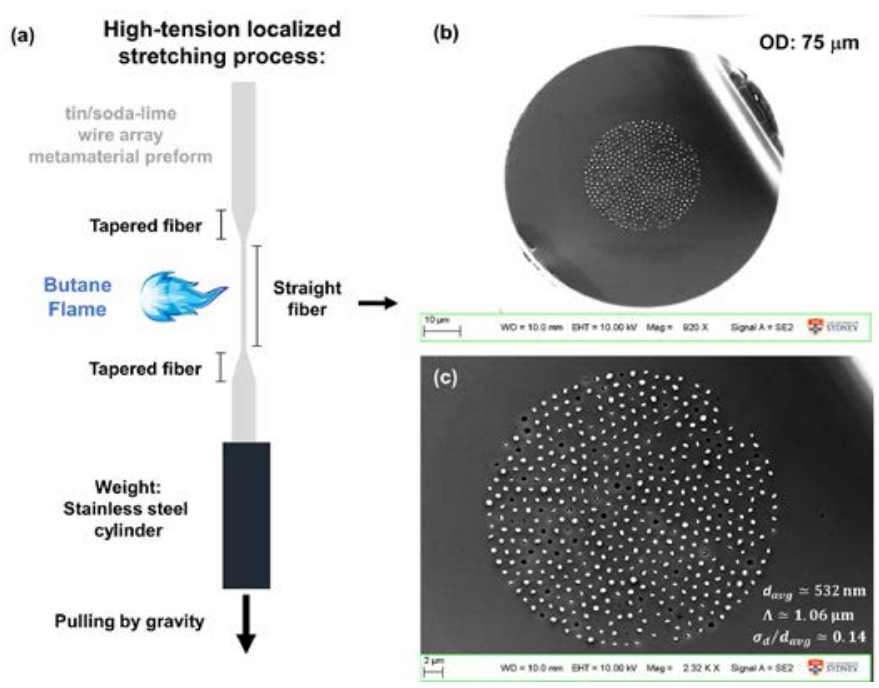

Fig. 5. (a) Schematic of the high-tension localized stretching process and the resultant samples: two tapered fibers (magnifying hyperlenses) and one straight/stretched fiber. (b) Final tin/soda-lime metamaterial fiber with $d_{\text {avg }} \simeq 532 \mathrm{~nm}, \Lambda \simeq 1.06 \mu \mathrm{m}, \quad \sigma_{d} / d_{\text {avg }} \simeq 0.14$, fabricated by stretching of the metamaterial preform of Fig. 3. (c) Detail of the wire array region of the straight/stretched metamaterial fiber.

region, and further study is required to quantify and characterized its influence on the instabilities.

In our high-tension localized stretching process, the metamaterial preform is locally heated by a butane torch while a weight previously attached to the lower end of the preform stretches the sample under gravity, as illustrated in Fig. 5(a). This process yields three samples: two approximately conical transition regions, called tapered metamaterial fibers (magnifying hyperlenses), and a straight fiber between them (Fig. 5(a)). Note that the straight region possesses a slight variation in the outside diameter along its length, which is not detrimental to applications that are limited to short samples. For imaging applications in the MIR, such wire array metamaterial fibers are naturally limited to samples with an overall length in the hundreds of micron scale due to their high optical losses caused by the presence of the metal [21].

Figure 5(b) and Fig. 5(c) show a cross-section micrograph of a tin/soda-lime metamaterial fiber (straight fiber) fabricated by the high-tension localized stretching process of the metamaterial preform (Fig. 3) using a pulling weight of $50 \mathrm{~g}$. Such weight generates an initial pulling tension per area of $63.6 \mathrm{~g} / \mathrm{mm}^{2}$ in the metamaterial preform ( $\mathrm{OD}=1 \mathrm{~mm}$ ) and a pulling tension per area of $11.32 \mathrm{~kg} / \mathrm{mm}^{2}$ in the final fiber ( $\mathrm{OD}=75 \mu \mathrm{m}$ ). The resultant structure obtained has $d_{\text {avg }} \simeq 532 \mathrm{~nm}, \Lambda \simeq 1.06 \mu \mathrm{m}$ and $\sigma_{d} / d_{\text {avg }} \simeq 0.14$, which represents a remarkable uniformity for such dimensions.

The new values of tension available with the high-tension localized stretching process and the influence of other favorable parameters (smaller dwell time, different neck-down profile) allow the fabrication of uniform wire array structures even with $d_{\text {avg }}<250 \mathrm{~nm}$. Figure 6(a) and Fig. 6(b) show two further examples of wire array metamaterial fibers with $\sigma_{d} / d_{\text {avg }} \quad$ smaller than $0.25, \quad d_{\text {avg }} \simeq 249 \mathrm{~nm}$ and $d_{\text {avg }} \simeq 143 \mathrm{~nm}$, respectively. Figure 6(c) shows the instability term $\sigma_{d} / d_{a v g}$ as a function of $d_{a v g}$ for tin/soda-lime wire array structures made by drawing at the limit of the pulling tension (red curve) and by the high-tension localized stretching process (green curve) in the last fabrication step.

The level of quality $\sigma_{d} / d_{\text {avg }}<0.2$ reached for wires with a diameter larger than $240 \mathrm{~nm}$ in the tin/soda-lime system can only be obtained with wire diameters $\simeq 6 \mu \mathrm{m}$ in the indium/PMMA system with continuous drawing (Fig. 4(c) and [12]). Therefore, it is clear that our replacement of the polymer with soft-glasses and the employment of the hightension localized stretching process in the last fabrication step allowed the fabrication of wire structures $25 \mathrm{x}$ smaller with an equivalent quality, which is a remarkable improvement in such a complex co-drawing process.

Besides its slightly higher fluctuation in the wire diameters ( $\sigma_{d} / d_{\text {avg }} \simeq 0.25$ ), the smallest structure fabricated by the high-tension localized stretching process (Fig. 6(b), $d_{\text {avg }} \simeq 143 \mathrm{~nm}$ ) can still be employed for imaging and lifetime engineering applications [21]. Comparing again with the instabilities seen in the indium/polymer system under continuous drawing (Fig. 4(c) and [12]), $\sigma_{d} / d_{\text {avg }}<0.25$ can be only achieved in the indium/polymer system with wires larger than $3.5 \mu \mathrm{m}$. Therefore, for this size of structure, the use of soft-glass and the optimization of the stretching process made possible the fabrication of a wire array structure $\simeq 24 \mathrm{x}$ smaller with equivalent quality.

Considering again the Plateau-Rayleigh instability limit as the wire diameter corresponding to $\sigma_{d} / d_{\text {avg }}=1 / e \simeq 0.37$, the extrapolation of our experimental data with the fitting in the form $\alpha e^{\beta / d}$ of Fig. 6(c) indicates that such limit for the fabricated tin/soda-lime structures shifted from $d_{\text {avg }} \simeq 550 \mathrm{~nm}$ in the drawing process to $d_{\text {avg }} \simeq 100 \mathrm{~nm}$ in the high-tension localized stretching process. This would represent a reduction of $\simeq 5.5 \mathrm{x}$ in the Plateau-Rayleigh instability limit due to the replacement of the final stretching process. Comparing with the instabilities seen in the indium/polymer system (Fig. 4(c) and [12]), our work suggests the replacement of the polymer for soft-glass and the employment of the high-tension localized stretching process could result in an overall reduction of $\simeq 18 \mathrm{x}$ in the PlateauRayleigh instability limit (from $d_{\text {avg }} \simeq 1.8 \mu \mathrm{m}$ to $\simeq 100 \mathrm{~nm}$ ).

The instability plot of Fig. 6(c) also suggests that this approach could fabricate relatively uniform wire array fibers with average wire diameter as small as $100 \mathrm{~nm}$, which are not in the scope of this work: With such wire sizes, metamaterials exhibit huge optical losses, making them unusable for imaging application at mid-infrared frequencies [21].

Finally, significant improvement in the high-tension localized stretching process can be achieved by adapting conventional tapering setups and techniques commonly used 

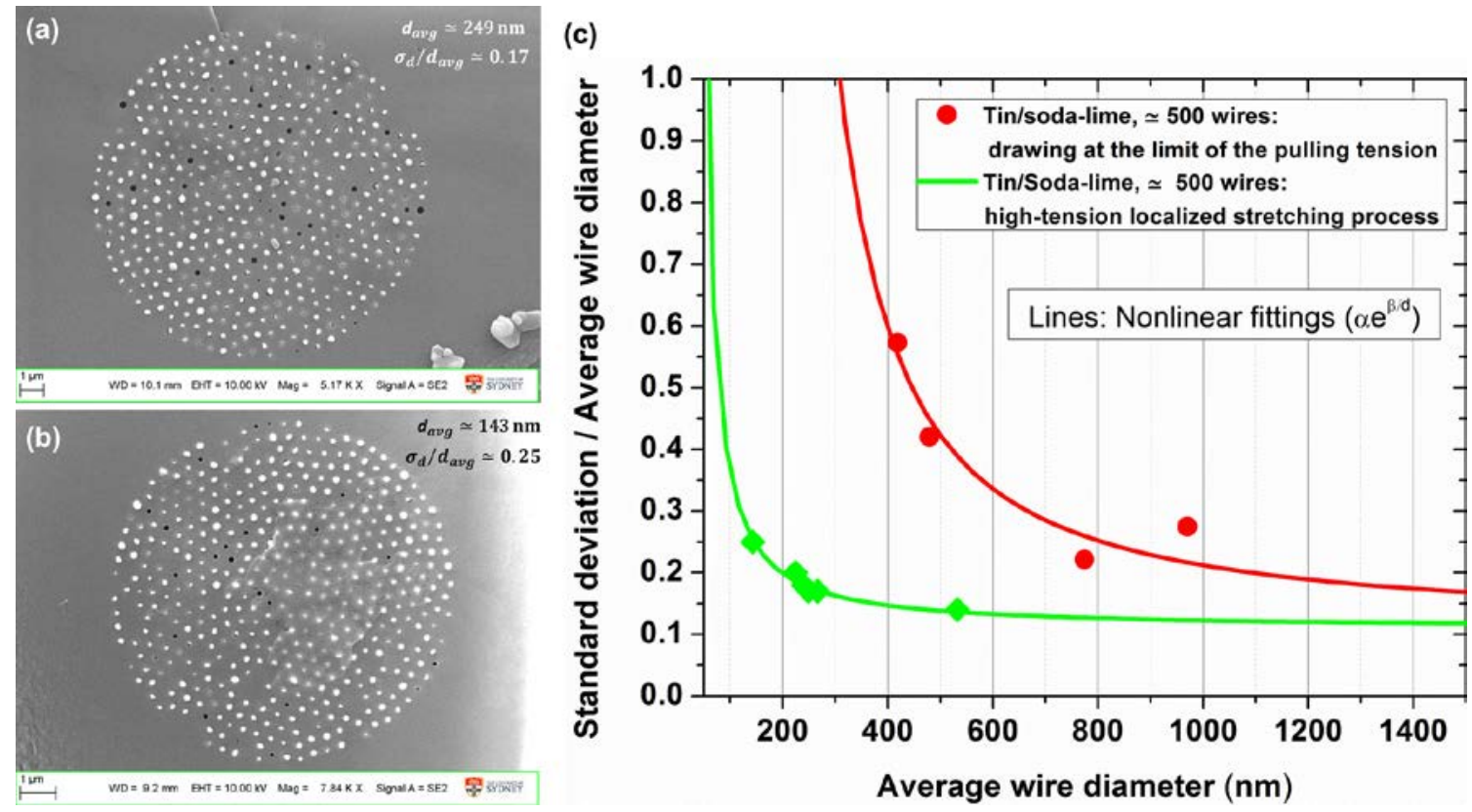

Fig. 6. Examples of tin/soda-lime wire array metamaterial fibers fabricated by the high-tension localized stretching process of the metamaterial preform showed in Fig. 3 ( $d_{\text {avg }} \simeq 9.34 \mu \mathrm{m}, \Lambda \simeq 18 \mu \mathrm{m}$ ): (a) $d_{\text {avg }} \simeq 249 \mathrm{~nm}, \Lambda \simeq 500 \mathrm{~nm}, \sigma_{d} / d_{\text {avg }} \simeq 0.17$; (b) $d_{\text {avg }} \simeq 143 \mathrm{~nm}, \Lambda \simeq 285 \mathrm{~nm}, \sigma_{d} / d_{\text {avg }} \simeq 0.25$. (c) Comparison of the instability term $\sigma_{d} / d_{\text {avg }}$ as a function of the $d_{\text {avg }}$ for tin/soda-lime wire array structures made by drawing at the limit of the pulling tension (red curve) and by the high-tension localized stretching process (green curve) for the last fabrication step. The curves correspond to nonlinear fittings of the function $\alpha e^{\beta / d}$, which is the expected form according to the standard deviation of (1) [12].

for optical fibers. Better control over important parameters such as the length of the hot zone, the pulling temperature and heating time could enable the fabrication of extremely steep tapers in short lengths, resulting in the fabrication of tapered magnifying hyperlenses for super-resolution imaging at midinfrared frequencies [34].

\section{CONCLUSION}

We have demonstrated the fabrication of tin/soda-lime wire array metamaterial fibers with highly uniform structures with average wire diameter as small as $143 \mathrm{~nm}$, compatible with operation at mid-infrared frequencies. The suppression of the Plateau-Rayleigh instability for such small structures was made possible by employing soda-lime glass as the dielectric medium and maximizing the viscosity of the glass during the drawing/stretching. Replacing the continuous drawing by a high-tension localized stretching process enabled a more than 5 fold reduction in achievable stable wire diameter. The established fabrication process represents a low-cost and large volume production alternative for hyperbolic metamaterials with operation wavelength as short as the mid-infrared. The newly drawn wire array metamaterial fibers and preforms offer the possibility to fabricate tapered samples, giving rise to wire array magnifying hyperlenses for subdiffraction imaging [5], [13], [34], in the MIR, which cannot practically be fabricated with conventional planar techniques.

\section{Acknowledgment}

Juliano Hayashi thanks the Science without Borders Program by CAPES (grant 9468/13-7). This project was performed in part at the Optofab node of the Australian National Fabrication Facility (ANFF) using Commonwealth and NSW State Government funding. This project has received funding from the European Union's Horizon 2020 research and innovation programme under the Marie Sklodowska-Curie grant agreement No. 708860. Simon Fleming acknowledges funding from ARC Discovery Project DP140104116.

The authors acknowledge the facilities and the scientific and technical assistance of the Australian Microscopy \& Microanalysis Research Facility at the Australian Centre for Microscopy \& Microanalysis at the University of Sydney.

\section{REFERENCES}

[1] T. J. Cui, D. R. Smith, and R. P. Liu, in Metamaterials, New York, NY, USA: Springer, 2010.

[2] N. Fang, and X. Zhang, "Imaging properties of a metamaterial superlens,” Appl. Phys. Lett., vol. 82, no. 2, pp. 161-163, Jan 2003.

[3] N. Yu, and F. Capasso, "Flat optics with designer metasurfaces,” Nat. Mater., vol. 13, pp. 139-150, Jan. 2014.

[4] Z. Jacob, L. V. Alekseyev, and E. Narimanov, “Optical Hyperlens: Far-field imaging beyond the diffraction limit," Opt. Express, vol. 14, no. 18, pp. 8247-8256, Sept. 2006.

[5] P. A. Belov, G. K. Palikaras, Y. Zhao, A. Rahman, C. R. Simovski, Y. Hao, and C. Parini, “Experimental demonstration of multiwire endoscopes capable of manipulating near-fields with subwavelength resolution," Appl. Phys. Lett., vol. 97, article no. 191905, Oct. 2010. 
[6] W. Cai, U. K. Chettiar, A. V. Kildishev, and V. M. Shalaev, "Optical cloaking with metamaterials," Nat. Photonics, vol. 1, no. 4, pp. 224-227, Apr. 2007.

[7] A. F. Abouraddy, M. Bayindir, G. Benoit, S. D. Hart, K. Kuriki, N. Orf, O. Shapira, F. Sorin, B. Temelkuran, and Y. Fink, "Towards multimaterial multifunctional fibres that see, hear, sense and communicate," Nat. Mater., vol. 6, no. 5, pp. 336-347, May 2007.

[8] G. Tao, A. M. Stolyarov, and A. F. Abouraddy, “Multimaterial fibers," Int. J. Appl. Glass Sci., vol. 3, no. 4, pp. 349-368, Nov. 2012.

[9] A. Tuniz, R. Lwin, A. Argyros, S. C. Fleming, E. M. Pogson, E. Constable, R. A. Lewis, and B. T. Kuhlmey, "Stacked-and-drawn metamaterials with magnetic resonances in the terahertz range," Opt. Express, vol. 19, no. 17, pp. 16480-16490, Aug. 2011.

[10] N. Singh, A. Tuniz, R. Lwin, S. Atakaramians, A. Argyros, S. C. Fleming, and B. T. Kuhlmey, "Fiber-drawn double split ring resonators in the terahertz range," Opt. Mater. Express, vol. 2, no. 9, pp. 1254-1259, Sept. 2012.

[11] A. Tuniz, B. T. Kuhlmey, R. Lwin, A. Wang, J. Anthony, R. Leonhardt, and S.C. Fleming, "Drawn metamaterials with plasmonic response at terahertz frequencies," Appl. Phys. Lett., vol. 96, article no. 191101, Apr. 2010.

[12] O. T. Naman, M. R. New-Tolley, R. Lwin, A. Tuniz, A. H. Al-Janabi, I. Karatchevtseva, S. C. Fleming, B. T. Kuhlmey, and A. Argyros, "Indefinite Media Based on Wire Array Metamaterials for the THz and Mid-IR," Adv. Opt. Mater., vol. 1, no. 12, pp. 971-977, Nov. 2013.

[13] A. Tuniz, K. J. Kaltenecker, B. M. Fischer, M. Walther, S.C. Fleming, A. Argyros, and B.T. Kuhlmey, "Metamaterial fibres for subdiffraction imaging and focusing at terahertz frequencies over optically long distances," Nat. Commun., vol. 4, article no. 2706, Oct. 2013.

[14] S. Tomotika, "On the instability of a cylindrical thread of a viscous liquid surrounded by another viscous fluid," Proc. $R$. Soc. Lond. A., vol. 150, no. 870, pp. 322-337, Jun. 1935.

[15] S. Tomotika, "Breaking up of a drop of viscous liquid immersed in another viscous fluid which is extending at a uniform rate," Proc. R. Soc. Lond. A., vol. 153, no. 879, pp. 302-318, Jan. 1936.

[16] S. Shabahang, J.J. Kaufman, D. S. Deng, and A. F. Abouraddy, "Observation of the Plateau-Rayleigh capillary instability in multi-material optical fibers," Appl. Phys. Lett., vol. 99, article no. 161909, Oct. 2011.

[17] A. Alchalaby, R. Lwin, A.H Al-Janabi, P. W. Trimby, S. C. Fleming, B. T. Kuhlmey, and A. Argyros, "Investigation of Plateau-Rayleigh Instability in Drawn Metal-Polymer Composite Fibers for Metamaterials Fabrication," $J$. Lightwave Technol., vol. 34, no. 9, pp. 2198-2205, May 2016.

[18] G. F. Taylor, "A method of drawing metallic filaments and a discussion of their properties and uses," Phys. Rev., vol. 23, no. 5, pp. 655-660, May 1924.

[19] X. Zhang, Z. Ma, Z. Y. Yuan, and M. Su, "Mass-productions of vertically aligned extremely long metallic micro/nanowires using fiber drawing nanomanufacturing,” Adv. Mat., vol. 20, no. 7, pp. 1310-1314, Mar. 2008.

[20] E. Badinter, A. Ioisher, E. Monaico, V. Postoloache, and I. M. Tiginyanu, "Exceptional integration of metal or semimetal nanowires in human-hair-like glass fiber," Mater. Lett., vol. 64, no. 17, pp. 1902-1904, Sept. 2010.

[21] J. G. Hayashi, S. Fleming, B. T. Kuhlmey, and A. Argyros, "Metal selection for wire array metamaterials for infrared frequencies," Opt. Express, vol. 23, no. 23, pp. 29867-29881, Nov. 2015.

[22] S. R. Choudhury, and Y. Jaluria, "Practical aspects in the drawing of an optical fiber," J. Mater. Res., vol. 13, no. 2, pp. 483-493, Feb. 1998.

[23] M. J. Assael, I. J. Armyra, J. Brillo, S. V. Stankus, J. Wu, and W. A. Wakeham, "Reference data for the density and viscosity of liquid cadmium, cobalt, gallium, indium, mercury, silicon, thallium, and zinc," J. Phys. Chem. Ref. Data, vol. 41, article no. 033101, May 2012.

[24] M. C. J. Large, L. Poladian, G. W. Barton, and M. A. Eijkelenborg, in Microstructured Polymer Optical Fibres, Springer Science \& Business Media, US, 2007.

[25] M. J. Assael, A. E. Kalyva, K. D. Antoniadis, R. M. Banish, I. Egry, J. Wu, E. Kaschnitz, and W. A. Wakeham, "Reference data for the density and viscosity of liquid copper and liquid tin,” J. Phys. Chem. Ref. Data, vol. 39, article no. 033105, Jun. 2010.

[26] Schott Technical Glasses, Physical and technical properties, general information datasheet, accessed: Oct. 2018, https://www.schott.com/d/uk/76a1e227-63ac-4f88-b111efd65c41c95e/1.0/schott_techn_glaeser_e_2007.pdf.

[27] T. Tanaka, M. Nakamoto, R. Oguni, J. Lee, and S. Hara, "Measurement of the surface tension of liquid $\mathrm{Ga}, \mathrm{Bi}$, Sn, In and $\mathrm{Pb}$ by the constrained drop method," Z. Metallkd., vol. 95, no. 9, pp. 818-822, Sept. 2004.

[28] J. Lee, W. Shimoda, and T. Tanaka, "Surface tension and its temperature coefficient of liquid $\mathrm{Sn}-\mathrm{X}(\mathrm{X}=\mathrm{Ag}, \mathrm{Cu})$ alloys," Mater. Trans., vol. 45, no. 9, pp. 2864-2870, Jul. 2004.

[29] B. B. Alchagirov, R. K. Dadashev, F. F. Dyshekova, and D. Z. Elimkhanov, "Temperature dependence of the surface tension of indium," Russ. J. Phys. Chem. A, vol. 87, no. 6, pp. 890-894, Jun. 2013.

[30] George F. Taylor, "Process and apparatus for making filaments,” U.S. Patent n. 1793529A, Feb. 24, 1931.

[31] J. D. Ayers, "Glass fibres with fine conducting cores," $J$. Mater. Sci., vol. 28, no. 9, pp. 2337-2346, May 1993.

[32] P. Russell, "Photonic crystal fibers," Science, vol. 299, no. 5605, pp. 358-362, Jan. 2003.

[33] J. C. Knight, "Photonic crystal fibres," Nature, vol. 424, no. 6950, pp. 847-851, Aug. 2003.

[34] J. G. Hayashi, "Wire array infrared metamaterial fibres: Fabrication and applications," Ph.D. dissertation, School of Physics, University of Sydney, Sydney, Australia, 2017.

[35] Schott AR-Glas technical data, accessed: Oct. 2018, https://www.schott.com/d/tubing/78e67f8d-8da6-48bb-8e8557c128530f05/schott-tubing-datasheet-ar-glas-english.pdf. 
[36] N. K. Goel, R. H. Stolen, S. Morgan, J. K. Kim, D. Kominsky, and G. Pickrell, "Core-suction technique for the fabrication of optical fiber preforms," Opt. Lett., vol. 31, no. 4, pp. 438-440, Feb. 2006.

[37] M. Fokine, L.E. Nilsson, Å. Claesson, D. Berlemont, L. Kjellberg, L. Krummenacher, and W. Margulis. "Integrated fiber Mach-Zehnder interferometer for electro-optic switching," Opt. Lett., vol. 27, no. 18, pp. 1643-1645, Sept. 2002. 